\title{
Un enseignement supérieur bousculé pour le meilleur peut-être...
}

\section{Christelle Lison}

\section{(2) OpenEdition \\ 1 Journals}

Electronic version

URL: https://journals.openedition.org/ripes/3309

DOI: 10.4000/ripes.3309

ISSN: 2076-8427

\section{Publisher}

Association internationale de pédagogie universitaire

\section{Electronic reference}

Christelle Lison, "Un enseignement supérieur bousculé pour le meilleur peut-être...", Revue internationale de pédagogie de l'enseignement supérieur [Online], 37(3) | 2021, Online since 15 November 2021,

connection on 17 January 2023. URL: http://journals.openedition.org/ripes/3309 ; DOI: https://doi.org/ 10.4000/ripes.3309

This text was automatically generated on 17 January 2023.

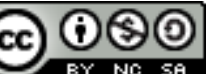

Creative Commons - Attribution-NonCommercial-ShareAlike 4.0 International - CC BY-NC-SA 4.0 https://creativecommons.org/licenses/by-nc-sa/4.0/ 


\title{
Un enseignement supérieur bousculé pour le meilleur peut- être...
}

\author{
Christelle Lison
}

1 L'année 2021 se termine. Elle aura été presque aussi particulière que 2020. Si aujourd'hui, l'activité reprend tranquillement, force est de constater que nos us et coutumes se sont modifiés. L'enseignement supérieur a vécu une crise majeure, mais aussi source de transformations positives. Nous verrons dans les prochains mois et dans les prochaines années comment les établissements d'enseignement supérieur et les acteurs qui y évoluent se saisissent des enjeux qui ont émergé au cours des 18 derniers mois, mais également des pistes de transformation qui ont été proposées, mises à l'essai voire intégrées tant par les étudiants que par les personnels administratifs et les professeurs. En parallèle les questions de recherche ont, elles aussi, évolué. Certaines se posent avec une nouvelle acuité, d'autres ont émergé. Cela ne se fait pas nécessairement facilement. Tel que je le mentionnais dans mon précédent édito, cela transforme également le monde de la publication, tant professionnelle que scientifique. Les acteurs se sont dernièrement fédérés dans des groupes parfois formels, mais souvent informels, leur permettant rapidement de partager des idées, des pratiques, des résultats de recherche. Cela pose forcément question, surtout dans un monde de la publication en sciences humaines et sociales où le service à la collectivité volontaire et bénévole est au cœur même du système. D'autres secteurs ont déjà transformé leurs pratiques, peut-être est-ce là une voie à explorer. Les prochaines années seront certainement propices à la réflexion, pour ne pas perdre ce que la crise a fait émerger de pertinents. En ce sens, le prochain colloque de l'AIPU (https:// www.aipu2022.fr/) sera un lieu d'effervescence pour voir comment l'" agir ensemble » constitue une clé de notre transformation collective. Offrir à la collectivité les résultats de sa recherche en est d'ailleurs un levier.

Pour le troisième numéro de l'année, nous vous proposons de découvrir neuf articles. 
3 Le premier article est celui de collègues belges, Marie-Claude Hublet, Sabine Lontie, Mohammed Arras, Sophie Remacle et Sylviane Bachy. Partant du constat d'un taux d'échec important en enseignement supérieur, ils proposent un article intitulé «Test diagnostique à l'entrée de l'enseignement supérieur : validation et usages » au cœur duquel ils analysent des tests diagnostiques de rentrée mis en place par le Service d'Accompagnement des Etudiants de la Haute Ecole Vinci-Site Parnasse-ISEI. Ceux-ci font partie des nombreux dispositifs d'aide à la réussite et à l'orientation et les résultats permettraient d'identifier les étudiants à risque, se voulant ainsi une base pour guider les enseignants dans la mise en place de nouvelles activités d'enseignement, pour guider les étudiants dans les choix personnels et leur engagement à participer aux activités de renforcement et dans le développement de dispositifs d'accompagnement.

4 Le deuxième article est celui de collègues québécois, Stéphane Thibodeau, Elisabeth Giguère, Sonia Lefebvre et Eric Frenette. Intitulé «Estime de soi, sentiment d'autoefficacité et intention de décrocher au collégial ", il aborde la délicate question du décrochage scolaire. L'objectif de leur recherche est de mettre en lumière des facteurs personnels sous-jacents au décrochage scolaire. À l'aides d'outils validés, ils ont interrogé 391 étudiants provenant de trois établissements collégiaux (deux publics et un privé). Les résultats montrent que l'estime de soi et le sentiment d'efficacité des étudiants du collégial présentent des corrélations négatives avec l'intention de décrocher. Plus précisément, l'analyse acheminatoire présente des statistiques d'ajustement adéquates du modèle aux données. Elle révèle l'existence de liens indirects entre l'estime de soi et l'intention de décrocher, de même que le rôle d'intermédiaire du sentiment d'autoefficacité dans ces relations.

5 Le troisième article est celui de collègues français, Najoua Mohib, Simon Zingaretti et Rémi Bachelet. Cat article, intitulé « Améliorer le sentiment de compétence en gestion de projet des étudiants : résultats d'une démarche SoTL ». Après avoir observé que les étudiants ne se sentaient pas prêts à conduire un projet à l'issue de leur master de sciences de l'éducation et de la formation à l'Université de Strasbourg, une nouvelle formule pédagogique a été mise en place. Afin d'en valider les retombées, les auteurs ont mené une recherche dans une perspective Scholarship of Teaching and Learning (SoTL) auprès des étudiants (questionnaire d'autoévaluation et entretien). Si les résultats montrent une augmentation significative du sentiment de compétence en gestion de projet à l'issue de l'expérimentation, il n'est toutefois pas possible d'établir de liens effectifs avec les méthodes pédagogiques mises en place.

6 Le quatrième article est celui de deux personnes du département de psychologie de l'Université du Québec à Montréal (Québec), Valérie Michaud et Georgette Goupil. Celui porte sur le « Trouble du spectre de l'autisme et études postsecondaires : points de vue d'intervenants des services d'aide aux étudiants ». Concrètement, leur étude qualitative a pour objectif de recueillir les expériences rapportées d'intervenants (14) des services d'aide d'établissements postsecondaires québécois quant aux forces et aux difficultés observées chez les étudiants ayant un trouble du spectre de l'autisme. Elle vise aussi la description des expériences rapportées de ces intervenants concernant les besoins perçus de soutien des professeurs et des autres acteurs du milieu. Les résultats soulèvent la complexité des demandes de soutien pouvant être associées trouble du spectre de l'autisme, les cibles d'intervention et le besoin de formation des personnels.

7 Le cinquième article porte sur « L'enseignement de la mécanique quantique grâce à un récit de fiction ». Fabrice Ferlin, Philippe Lautesse, Juliette Tuaillon, Jean-Loup Héraud 
et Lionel Chaussard, de l'Université Claude Bernard Lyon 1 (France) proposent, dans cet article, d'introduire un questionnement épistémologique sur les concepts physiques mis en jeu dans la mécanique quantique en mettant l'accent sur le problème de la mesure. Pour ce faire, ils s'emparent de ce questionnement au travers d'un récit de fiction réaliste, le roman «Isolation» de Greg Egan. Ils mobilisent alors un cadre théorique, issu des travaux du philosophe David Lewis, en vue de montrer la pertinence de ce roman pour une utilisation dans un cours de mécanique quantique en deuxième année de licence de physique.

8 Le sixième article, « Analyse des freins à l'adoption des SPOC en classes inversées ", est celui de Narjes Sassi et Caroline Cloonan. Alors que les classes inversées sont de plus en plus mises en place dans les établissements d'enseignement supérieur, les autrices interrogent le problème de leur adoption par l'équipe enseignante et celui de leur acceptation par les apprenants. Elles analysent dès lors ces freins grâce aux retours d'expériences de responsables pédagogiques au sein d'une école de commerce qui ont mis en œuvre et suivi des dispositifs de classes inversées avec usage de Small Private Online Course (SPOC). Chez les enseignants, les principaux freins sont liés à leurs rôles, aux continus, au suivi des travaux en ligne et aux difficultés en lien avec l'évaluation. Chez les apprenants, les freins sont de l'ordre de l'organisation et de l'autonomie du travail, de la non-immédiateté d'interaction avec l'enseignant et du manque de compréhension de la pertinence de cette modalité pédagogique.

9 Le septième article est celui de deux collègues de l'Université de Sherbrooke (Québec), Josée Lachance et Jean-François Desbiens. Intitulé «Collaboration et construction identitaire d'une étudiante chercheuse au doctorat en éducation », il touche un objet bien particulier. En effet, l'article relate le chemin parcouru par une doctorante durant sa formation encadrée par une équipe formée d'un directeur et de deux directeurs adjoints afin de retracer les étapes importantes et les caractéristiques de la collaboration. L'article met en lumière les conditions qui ont favorisé le point de bascule vers l'autonomie tel qu'il a été vécu par l'étudiante, ce qui a mené à sa construction identitaire comme chercheuse. Ainsi, la construction de la confiance et le respect ont été des conditions essentielles. De plus, les moments de crise ont été gérés à partir d'une médiation entre le pôle de supervision et d'accompagnement habité par différents membres de l'équipe.

10 Le huitième article de ce dernier numéro 2021 est également proposé par deux collègues de l'Université de Sherbrooke (Québec), Mélanie Cabana et Denis Bédard. Intitulé «Microprogramme en pédagogie de l'enseignement supérieur pour les enseignants : incidences sur la motivation et les stratégies d'apprentissage des étudiants ", il tente de mesurer les incidences de ce programme de formation sur la motivation et les stratégies d'apprentissage des étudiants ayant suivi les cours d'enseignants formés au MPES. Les données, recueillies auprès de 252 étudiants inscrits dans six programmes de formation différents, montrent que les enseignants formés ont proposé des exposés et des activités favorisant le maintien de la motivation des étudiants tout au long du semestre et la mobilisation d'une diversité de stratégies d'apprentissage variées.

11 Le dernier article porte sur la « Formation interdisciplinaire à la pédagogie dans l'enseignement supérieur : de l'expérience individuelle à la dynamique collective pour impulser une transformation pédagogique ». Proposé par des collègues françaises, Catherine de la Fouchardière, Laure Abiad, Moussa Laanani, Marine Lanteri, Morgane 
Maridet, Annabelle Tenenbaum, Isabelle Colombet, il est le fruit d'une collaboration réflexive d'autrices issues de champs disciplinaires et de structures universitaires différents. Il s'agit d'un retour d'expérience à la suite du parcours CertifiENS afin de mettre en lumière ce que celui-ci a permis aux participantes de réaliser en termes de transformation pédagogique, et ce, grâce à l'accompagnement de conseillères pédagogiques. Leur analyse montre que l'interdisciplinarité a été un atout majeur et une source de synergies, permettant de s'intéresser spécifiquement à la pédagogie et de penser un changement de paradigme dans la durée. 